\section{Cuestionario de prácticas pedagógicas: análisis de su estructura factorial y consistencia interna en docentes de carreras de la salud}

\author{
CRISTHIAN PÉREZ V. ${ }^{1, a}$, GIULIETTA VACCAREZZA G. ${ }^{2, \mathrm{~b}}$, \\ CÉSAR AGUILAR A. ${ }^{3, \mathrm{c}}$, KATHERINE COLOMA N., ${ }^{4, \mathrm{~d}}$, \\ HORACIO SALGADO F. ${ }^{2, \mathrm{e}}$, MARJORIE BAQUEDANO R. ${ }^{5, \mathrm{f}}$, \\ CARLA CHAVARRÍA R. ${ }^{6, g}$, NANCY BASTÍAS V., h
}

\section{Factor analysis and internal consistency of pedagogical practices questionnaire among health care teachers}

Background: Teaching practice is one of the most complex topics of the training process in medicine and other health care careers. The Teaching Practices Questionnaire (TPQ) evaluates teaching skills. Aim: To assess the factor structure and internal consistency of the Spanish version of the TPP among health care teachers. Material and Methods: The TPQ was answered by 315 university teachers from 13 of the 15 administrative Chilean regions, who were selected through a non-probabilistic volunteer sampling. The internal consistency of TPP factors was calculated and the correlation between them was analyzed. Results: Six factors were identified: Student-centered teaching, Teaching planning, Assessment process, Dialogue relationship, Teacher-centered teaching and Use of technological resources. They had Cronbach alphas ranging from 0.60 to 0.85 . Conclusions: The factorial structure of $T P Q$ differentiates the most important functions of teaching. It also shows a theoretical consistency and a practical relevance to perform a diagnosis and continuous evaluation of teaching practices. Additionally, it has an adequate internal consistency. Thus, TPQ is valid and reliable to evaluate pedagogical practices in health care careers.

(Rev Med Chile 2016; 144: 795-805)

Key words: Education, Medical; Educational Measurement; Faculty, Medical; Teaching.
'Departamento de Educación Médica, Facultad de Medicina, Universidad de Concepción, Concepción, Chile. ${ }^{2}$ Carrera de Psicología Sede Concepción, Facultad de Psicología, Universidad San Sebastián,

Concepción, Chile.

${ }^{3}$ Unidad de Investigación, Enfoque Émico, Concepción, Chile.

${ }^{4}$ Centro de Desarrollo de la Docencia, Universidad del Desarrollo, Concepción, Chile. ${ }^{5}$ Department of Sociology, University of Sheffield, Sheffield, Reino Unido. ${ }^{6}$ Facultad de Educación, Ciencias Sociales y Humanidades,

Universidad de La Frontera, Temuco, Chile.

aPsicólogo, Magíster en Psicología.

bsicóloga, Magíster en Ciencias de la Educación.

IIngeniero en Ejecución. dProfesora Básica, Magíster en Ciencias de la Educación.

ePsicólogo, Magíster en Psicología Comunitaria.

'Socióloga, Magíster en Investigación Social y Desarrollo.

9Profesora, Doctora (c) en Ciencias de la Educación.

hEnfermera, Magíster en Educación Médica para Ciencias de la Salud.

Trabajo financiado por el proyecto FONDECYT Regular 1110484.

Recibido el 26 de octubre de 2015 , aceptado el 31 de marzo de 2016.

\section{Correspondencia a:}

Cristhian Pérez Villalobos

Departamento de Educación Médica, Facultad de Medicina,

Universidad de Concepción.

Víctor Lamas 1290, Concepción,

Chile.

Teléfono: +56412204932

cperezv@udec.cl
$\mathrm{M}$ edicina y otras carreras de la salud son tensionadas por la globalización ${ }^{1}$, el mercado ${ }^{1-3}$, el desarrollo científico y tecnológico de la disciplina ${ }^{4}$, los cambios demográficos y sanitarios ${ }^{4}$ y las variaciones en la concepción de educación universitaria, que ha transitado desde un modelo centrado en la transmisión de conocimientos a uno orientado al desarrollo de profesionales reflexivos y autónomos ${ }^{4-6}$.
Las agendas nacionales e internacionales apremian a las carreras de la salud a mejorar sus procesos formativos para enfrentar estas presiones $^{4}$, compeliéndolas a revisar sus currículum ${ }^{7}$, desarrollar nuevos modelos de enseñanza ${ }^{3,8}, y$, principalmente, atender a las competencias de quienes implementan éstas: los docentes. Esto, pues la docencia se ha convertido en un tema central de discusión sobre la situación universita- 
ria $^{9-12}$, transformándose en un criterio de calidad de sus instituciones ${ }^{11}$. Dicha discusión no siempre contempla la complejidad del rol docente, que involucra diversas funciones: investigación, gestión, asistencia técnica, docencia, etc. ${ }^{13,14}$. Esta heterogeneidad de labores, fue sintetizada por Tejedor en cuatro grandes grupos: gestor de aprendizajes, facilitador, tutor y evaluador ${ }^{11}$, y por Harden en sus clásicos 12 roles del docente de educación médica ${ }^{15}$.

Aunque la función investigadora suele ser la que concentra las tres $\mathrm{R}$ (recursos, recompensas y reconocimiento) ${ }^{16}$, ésta y la calidad de especialistas de los docentes no son tan cuestionadas en la actualidad, como su capacidad pedagógica ${ }^{12}$. Sobre todo en Latinoamérica, cuyas universidades se caracterizan por una enseñanza expositiva y centrada en la transmisión de información ${ }^{17}$.

Actualmente, existe una preocupación consensuada por las prácticas pedagógicas de estos docentes, entendidas como los procesos donde éstos reflexionan y analizan los aspectos intelectuales y afectivos de las situaciones formativas, para definir cómo implementarán la enseñanza ${ }^{18,19}$. Estos procesos, complejos e intencionales, consideran las percepciones, significados y acciones de los actores involucrados ${ }^{19}$, y son afectados por el currículum oficial y el oculto ${ }^{19-21}$, involucrando aspectos culturales, políticos, sociales y económicos ${ }^{22}$.

La complejidad de las prácticas pedagógicas, problema ignorado por años pero urgente en la actualidad, ha impulsado a capacitar a los docentes universitarios en temáticas educativas ${ }^{10,23-26}$. Empero, estas instancias formativas suelen definirse desde la intencionalidad formativa de las universidades, pero no desde diagnósticos de la práctica docente. Lo anterior deriva, en parte, de la escasez de instrumentos para evaluar dichas prácticas, pues las evaluaciones docentes en las universidades son respondidas por estudiantes y están afectas a sesgos de respuesta ${ }^{27}$, y los instrumentos aplicados a académicos, ya sea sobre estilos de enseñanza ${ }^{28}$ o calidad docente ${ }^{29}$, no presentan evidencia de validez en población chilena.

Por ello, este equipo de investigación diseñó el Cuestionario de Prácticas Pedagógicas (CPP), con base en los resultados descriptivos de la fase cualitativa de este proyecto, respecto de las prácticas pedagógicas en docentes universitarios de Chile $^{26}$. Luego, el CPP fue sometido a evaluación de 15 de expertos en docencia universitaria, edu- cación, psicología educacional, educación médica y psicometría, y tuvo una aplicación piloto en 20 docentes de diversas disciplinas. Este instrumento, de auto-aplicación, permite describir la frecuencia con que los docentes universitarios, de cualquier área del conocimiento, realizan distintas actividades propias del quehacer formativo, a fin de diferenciar aquellas actividades tradicionales y centradas en el docente, de aquellas constructivistas centradas en el estudiante $e^{15,30}$.

El presente trabajo busca aportar evidencias de la validez y confiabilidad del CPP para la medición de las prácticas pedagógicas en docentes de carreras de la salud. Para esto, se analiza su validez de constructo evaluando la consistencia teórica de su estructura factorial, y su confiabilidad, analizando la precisión de sus mediciones ${ }^{31}$.

\section{Método}

\section{Participantes}

Mediante muestreo no probabilístico por voluntarios, se accedió a 315 profesionales que impartían docencia de pregrado en carreras de la salud en universidades chilenas, tanto tradicionales (adscritas al Consejo de Rectores de Universidades Chilenas, CRUCH) y privadas (no adscritas al CRUCH). Éstos residían en 13 de las 15 regiones geográficas del país (exceptuando las regiones de Atacama y Aysén), y dos residían en el extranjero.

\section{Instrumento}

Los docentes respondieron el Cuestionario de Prácticas Pedagógicas (CPP), que mide la frecuencia con que los docentes realizan conductas propias de su actividad docente, incluyendo actividades de planificación, enseñanza, evaluación, uso de recursos y gestión del ambiente educativo.

Presenta 60 afirmaciones sobre estas conductas, ante las cuales el participante debe responder según la frecuencia con que las ha realizado en los últimos dos años académicos, eligiendo una alternativa en escala tipo Likert de cinco alternativas $(1=$ nunca $o$ casi nunca; $2=$ rara vez; $3=$ a veces; $4=$ frecuentemente; $5=$ siempre o casi siempre).

Junto a este cuestionario, se aplicó un cuestionario sociodemográfico para describir la muestra.

\section{Procedimiento}

El cuestionario fue enviado a los docentes participantes a través de un correo electrónico en 
el que se les explicabas el propósito del proyecto, la participación solicitada y los antecedentes del investigador responsable. Debían acceder a un hipervínculo en el correo para ingresar al cuestionario, implementado en una plataforma de encuesta en línea. La primera página presentaba un consentimiento informado que los participantes debían aceptar para continuar con el cuestionario. Quince personas contactaron al investigador telefónicamente solicitando más antecedentes del estudio.

El procedimiento contó con la aprobación de la Comisión Nacional de Investigación Científica y Tecnológica de Chile, CONICYT.

\section{Análisis de datos}

Para evaluar la estructura factorial del CPP se empleó Análisis Factorial Exploratorio (AFE), aplicando Análisis de Ejes Principales, AEP, como método de extracción ${ }^{31,32}$.

Luego, se empleó el coeficiente alfa de Cron- bach para evaluar la confiabilidad de los factores detectados.

Por último, se realizó un análisis descriptivo de sus puntuaciones y se analizó su correlación mediante el coeficiente de Pearson.

Un valor de $\mathrm{p}<0,05$ fue considerado estadísticamente significativo. Se empleó STATA SE 11.0 para procesar los datos.

\section{Resultados}

De los 315 encuestados, se eliminó a quienes presentaban omisiones en el cuestionario analizado, obteniéndose una muestra válida de 296 académicos (Tabla 1).

El análisis inició evaluando la pertinencia del AFE, obteniéndose un estadístico de adecuación muestral Kaiser-Meyer-Olkin (KMO) de 0,89 y una prueba de esfericidad de Barlett estadísticamente significativa, $\chi^{2}(1770)=7.144,50 ; \mathrm{p}<0,001$, que apoyaron la realización del AFE.

\section{Tabla 1. Caracterización de la muestra de docentes de carreras de la salud}

\begin{tabular}{|c|c|c|}
\hline Variable & \multicolumn{2}{|l|}{ Estadísticos descriptivos } \\
\hline Edad (en años) & \multicolumn{2}{|c|}{$M=45,56 ;$ D.E $.=11,96 ;$ Mín = 24; Máx $=73$} \\
\hline Sexo & \multicolumn{2}{|l|}{$\begin{array}{l}\text { Hombre }(n=108 ; 36,49 \%) \\
\text { Mujer }(n=188 ; 63,51 \%)\end{array}$} \\
\hline Región de residencia & $\begin{array}{l}\text { Arica y Parinacota } \\
\text { Tarapacá } \\
\text { Antofagasta } \\
\text { Coquimbo } \\
\text { Valparaíso } \\
\text { Metropolitana } \\
\text { Libertador Gral. Bernardo O’Higgins } \\
\text { Maule } \\
\text { Bío Bío } \\
\text { Araucanía } \\
\text { Los Ríos } \\
\text { Los Lagos } \\
\text { Magallanes y la Antártica Chilena } \\
\text { Extranjero }\end{array}$ & $\begin{array}{l}(\mathrm{n}=5 ; 1,69 \%) \\
(\mathrm{n}=3 ; 1,01 \%) \\
(\mathrm{n}=9 ; 3,04 \%) \\
(\mathrm{n}=1 ; 0,34 \%) \\
(\mathrm{n}=18 ; 6,08 \%) \\
(\mathrm{n}=59 ; 19,93 \%) \\
(\mathrm{n}=1 ; 0,34 \%) \\
(\mathrm{n}=17 ; 5,74 \%) \\
(\mathrm{n}=127 ; 42,91 \%) \\
(\mathrm{n}=21 ; 7,09 \%) \\
(\mathrm{n}=20 ; 6,76 \%) \\
(\mathrm{n}=10 ; 3,38 \%) \\
(\mathrm{n}=3 ; 1,01 \%) \\
(\mathrm{n}=2 ; 0,68 \%)\end{array}$ \\
\hline $\begin{array}{l}\text { Ejercicio de la docencia en pregrado en el área de la } \\
\text { salud }\end{array}$ & $\begin{array}{l}\text { Como área principal } \\
\text { Como área secundaria }\end{array}$ & $\begin{array}{l}(n=244 ; 82,43 \%) \\
(n=52 ; 17,57 \%)\end{array}$ \\
\hline $\begin{array}{l}\text { Universidades donde ha dictado docencia en los últimos } \\
\text { cinco años (en número) }\end{array}$ & \multicolumn{2}{|l|}{$M=1,68 ; D . E .=1,03 ;$ Mín = 1; Máx = 7} \\
\hline $\begin{array}{l}\text { Ejercicio de docencia de pregrado en carreras de la salud } \\
\text { en los últimos } 5 \text { años }\end{array}$ & $\begin{array}{l}\text { En universidades adscritas al CRUCH } \\
\text { En universidades no adscritas al } \mathrm{CRUCH}\end{array}$ & $\begin{array}{l}(n=243 ; 82,09 \%) \\
(n=128 ; 43,24 \%)\end{array}$ \\
\hline Docentes con contrato indefinido & $\begin{array}{l}\text { En universidades adscritas al } \mathrm{CRUCH} \\
\text { En universidades no adscritas al } \mathrm{CRUCH}\end{array}$ & $\begin{array}{l}(\mathrm{n}=172 ; 58,11 \%) \\
(\mathrm{n}=50 ; 16,89 \%)\end{array}$ \\
\hline
\end{tabular}

$\mathrm{N}=$ 296; $\mathrm{M}=$ Media aritmética; D.E. = Desviación estándar; Mín = Mínimo; Máx = Máximo; $\mathrm{n}=$ Frecuencia absoluta. 
Posteriormente, se procedió a estimar el número de factores según dos criterios: Kaiser-Guttman o de raíz latente ${ }^{33}$ y Análisis Paralelo de Horn ${ }^{31}$. El primero identificó siete factores con valores propios (eigenvalues) mayores a $1,0(13,23,3,10$, $2,07,1,45,1,20,1,07$ y 1,02), explicando $80,04 \%$ de la varianza total de los ítems. El Análisis Paralelo, considerando 5.000 muestras aleatorias, también identificó siete factores con valores propios mayores a los presentados por $95 \%$ de las muestras aleatorias $(1,15,1,09,1,07,0,96,0,90,0,85$ y 0,81$)$.

Dada la convergencia entre criterios, se procedió a evaluar la distribución de los ítems en los siete factores, aplicando AEP con rotación oblicua Oblimin directo ${ }^{28}$. Al hacerlo, cinco ítems mostraron cargas factoriales inferiores al umbral de 0,30, propuesto como el mínimo adecuado para considerar significativa una carga factorial ${ }^{30}$. Por ello, se decidió eliminar el ítem que presentaba las cargas más bajas (ítem 4, "Incorporo explícitamente las contribuciones de los estudiantes durante las actividades"), y repetir el análisis con los 59 ítems remanentes. Bajo la misma estrategia, se debió eliminar progresivamente los ítems 15 ("Utilizo en su totalidad la hora de clases en actividades propias de la asignatura"), 41 ("Destaco los quiebres temáticos para pasar de un tema a otro"), 51 ("Reviso la pertinencia del programa de asignatura una vez finalizada ésta"), 56 ("Cuando tengo alguna dificultad con un estudiante la resuelvo de forma inmediata"), 55 ("Modifico la planificación de mis asignaturas cuando se requiere"), 26 ("Dejo claras cuáles serán las instancias de resolución de problemas desde el inicio del curso"), 25 ("Utilizo evaluaciones sumativas al cierre del semestre, tales como exámenes orales o escritos") y 52 ("Retroalimento a los estudiantes luego de sus intervenciones en clases").

Para los 51 ítems restantes se obtuvo un estadístico KMO de 0,89 con una prueba de Barlett, estadísticamente significativa, $\chi^{2}(1275)=5955,75$; $\mathrm{p}<0,001$, mostrando la viabilidad del AFE.

El criterio de Kaiser-Guttman identificó seis factores con valores propios sobre $1,0(11,52,2,71$, $1,98,1,38,1,13$ y 1,02 ), explicando $82,43 \%$ de la varianza total de los ítems. El Análisis Paralelo, considerando 5.000 muestras aleatorias, identificó seis factores con valores propios superiores a los presentados por $95 \%$ de las muestras aleatorias $(1,10,1,04,0,95,0,92,0,80$ y 0,73$)$.

A partir de eso, se evaluó la distribución de los ítems en los seis factores, mediante una matriz de configuración, empleando AEP con rotación oblicua Oblimin directo (Tabla 2). Todos los

\section{Tabla 2. Matriz de configuración del Cuestionario de Prácticas Pedagógicas en docentes de carreras de la salud obtenida mediante Análisis de Eje Principal con rotación Oblimin directo}

\begin{tabular}{|c|c|c|c|c|c|c|c|c|}
\hline Ítem & Reactivo & $\mathbf{I}$ & II & III & IV & $\mathbf{v}$ & VI & Comunalidad \\
\hline 1 & $\begin{array}{l}\text { Empleo sistemas de planificación para la docencia } \\
\text { tales como: calendarizaciones, syllabus, planificación } \\
\text { semanal, etc. }\end{array}$ & 0,036 & $0,606^{a}$ & 0,054 & $-0,083$ & 0,014 & 0,118 & 0,568 \\
\hline 2 & $\begin{array}{l}\text { Realizo evaluaciones de los avances que van teniendo } \\
\text { los alumnos }\end{array}$ & 0,217 & 0,393 & 0,033 & $-0,072$ & $-0,053$ & 0,120 & 0,712 \\
\hline 3 & $\begin{array}{l}\text { Recurro a las estrategias expositivas como primer op- } \\
\text { ción para enseñarle a mis estudiantes }\end{array}$ & 0,041 & $-0,118$ & $-0,174$ & $-0,003$ & 0,634 & 0,054 & 0,545 \\
\hline 5 & $\begin{array}{l}\text { Retroalimento detalladamente el desempeño de los } \\
\text { estudiantes luego de cada evaluación }\end{array}$ & 0,135 & 0,438 & 0,123 & 0,149 & $-0,045$ & $-0,089$ & 0,610 \\
\hline 6 & $\begin{array}{l}\text { Aplico evaluaciones escritas (p.e. certámenes, exáme- } \\
\text { nes, test, etc.) de respuesta cerrada como verdadero o } \\
\text { falso, o alternativas }\end{array}$ & 0,005 & 0,088 & 0,051 & $-0,075$ & 0,406 & 0,069 & 0,800 \\
\hline 7 & $\begin{array}{l}\text { En mis actividades académicas uso de los conoci- } \\
\text { mientos previos de los estudiantes como recursos de } \\
\text { enseñanza }\end{array}$ & 0,270 & 0,311 & 0,038 & 0,071 & $-0,024$ & $-0,041$ & 0,730 \\
\hline 8 & $\begin{array}{l}\text { Reviso la planificación de mis actividades (clases, super- } \\
\text { visiones, etc.) para analizar el avance alcanzado }\end{array}$ & 0,106 & 0,656 & 0,053 & 0,003 & $-0,012$ & 0,067 & 0,451 \\
\hline 9 & $\begin{array}{l}\text { Diseño situaciones de evaluación donde el estudiante } \\
\text { debe realizar conductas similares a las que se le pedi- } \\
\text { rán en su futuro laboral }\end{array}$ & 0,375 & 0,159 & 0,125 & $-0,113$ & $-0,001$ & 0,141 & 0,722 \\
\hline
\end{tabular}


Tabla 2. Continuación

\begin{tabular}{|c|c|c|c|c|c|c|c|c|}
\hline Ítem & Reactivo & I & II & III & IV & $\mathbf{v}$ & VI & Comunalidad \\
\hline 10 & $\begin{array}{l}\text { Evito el uso de herramientas tecnológicas (como } \\
\text { dispositivas, tecleras, videos, etc.) en mis actividades } \\
\text { docentes }^{(i)}\end{array}$ & $-0,025$ & 0,097 & 0,136 & $-0,026$ & 0,091 & $-0,474$ & 0,764 \\
\hline 11 & $\begin{array}{l}\text { Uso estrategias expositivas para introducir a los estu- } \\
\text { diantes a temas nuevos }\end{array}$ & 0,121 & 0,012 & $-0,202$ & $-0,052$ & 0,664 & 0,001 & 0,496 \\
\hline 12 & $\begin{array}{l}\text { Genero instancias para que los estudiantes se evalúen } \\
\text { entre ellos (coevaluación) }\end{array}$ & 0,003 & $-0,015$ & 0,793 & $-0,048$ & $-0,044$ & $-0,093$ & 0,390 \\
\hline 13 & $\begin{array}{l}\text { Cierro cada actividad docente (clases, supervisiones, } \\
\text { etc.) con un resumen de los contenidos o procedi- } \\
\text { mientos revisados }\end{array}$ & 0,030 & 0,375 & 0,221 & 0,255 & $-0,110$ & 0,026 & 0,562 \\
\hline 14 & $\begin{array}{l}\text { Al comienzo de cada actividad docente (clases, super- } \\
\text { visiones, etc.) presento los objetivos a los estudiantes }\end{array}$ & $-0,068$ & 0,453 & 0,154 & 0,170 & $-0,133$ & 0,074 & 0,633 \\
\hline 16 & $\begin{array}{l}\text { Organizo los contenidos o procedimientos de mis acti- } \\
\text { vidades docentes (clases, supervisiones, etc.) siguiendo } \\
\text { una lógica definida }\end{array}$ & $-0,012$ & 0,482 & $-0,097$ & 0,103 & 0,229 & 0,159 & 0,611 \\
\hline 17 & $\begin{array}{l}\text { Realizo una conexión entre los contenidos que enseño } \\
\text { y lo que los estudiantes verán en asignaturas futuras } \\
\text { del plan de estudios }\end{array}$ & 0,455 & 0,308 & $-0,073$ & 0,002 & 0,141 & 0,037 & 0,580 \\
\hline 18 & $\begin{array}{l}\text { Recomiendo el uso de herramientas tecnológicas a mis } \\
\text { estudiantes para la búsqueda de información }\end{array}$ & 0,127 & 0,175 & 0,032 & 0,059 & 0,038 & 0,383 & 0,708 \\
\hline 19 & $\begin{array}{l}\text { Hago preguntas amplias, abiertas, de respuestas varia- } \\
\text { das a los estudiantes para promover su participación }\end{array}$ & 0,431 & $-0,019$ & 0,183 & 0,119 & 0,001 & 0,159 & 0,620 \\
\hline 20 & $\begin{array}{l}\text { Planifico mis actividades docentes (clases, supervi- } \\
\text { siones, etc.) con anterioridad al inicio del período } \\
\text { académico }\end{array}$ & 0,046 & 0,560 & $-0,002$ & 0,084 & 0,146 & $-0,092$ & 0,601 \\
\hline 21 & $\begin{array}{l}\text { Doy espacios para que los estudiantes consulten } \\
\text { abiertamente }\end{array}$ & 0,173 & 0,222 & $-0,085$ & 0,337 & 0,071 & 0,052 & 0,696 \\
\hline 22 & $\begin{array}{l}\text { Reviso los contenidos que las asignaturas anteriores de } \\
\text { la malla han abordado para verificar que debo enseñar } \\
\text { en la mía }\end{array}$ & 0,278 & 0,336 & 0,257 & $-0,188$ & 0,175 & $-0,064$ & 0,593 \\
\hline 23 & $\begin{array}{l}\text { Uso la tecnología de la información para comunicarme } \\
\text { con mis estudiantes, como plataformas virtuales, sitios } \\
\text { web, etc. }\end{array}$ & 0,021 & 0,223 & 0,216 & 0,011 & 0,041 & 0,355 & 0,675 \\
\hline 24 & $\begin{array}{l}\text { Pido a mis estudiantes que usen tecnología en sus } \\
\text { presentaciones orales }\end{array}$ & $-0,025$ & 0,081 & 0,114 & 0,034 & 0,186 & 0,481 & 0,658 \\
\hline 27 & $\begin{array}{l}\text { Aplico instrumentos para que cada estudiante se eva- } \\
\text { lúen a sí mismo (autoevaluación) }\end{array}$ & 0,075 & 0,073 & 0,717 & $-0,062$ & $-0,036$ & $-0,054$ & 0,421 \\
\hline 28 & $\begin{array}{l}\text { Vinculo los contenidos que enseño con otras asignatu- } \\
\text { ras de la malla a través de ejemplos concretos }\end{array}$ & 0,672 & 0,129 & 0,028 & $-0,131$ & 0,059 & 0,051 & 0,489 \\
\hline 29 & $\begin{array}{l}\text { Hago preguntas de aplicación de contenidos que per- } \\
\text { mitan monitorear lo que los estudiantes han aprendido }\end{array}$ & 0,501 & 0,152 & $-0,027$ & $-0,074$ & 0,042 & 0,202 & 0,619 \\
\hline 30 & $\begin{array}{l}\text { Desarrollo actividades cooperativas en la sala de clase, } \\
\text { por ejemplo: trabajos de grupo, trabajo de pares, etc. }\end{array}$ & 0,255 & $-0,013$ & 0,472 & $-0,059-$ & $-0,124$ & 0,283 & 0,536 \\
\hline 31 & $\begin{array}{l}\text { Adecúo previamente el ambiente de la sala o lugar } \\
\text { de trabajo según las actividades que se van a realizar }\end{array}$ & 0,044 & 0,169 & 0,347 & 0,166 & $-0,126$ & 0,018 & 0,690 \\
\hline 32 & $\begin{array}{l}\text { Realizo actividades en las que los estudiantes deben } \\
\text { simular la aplicación de los contenidos tratados }\end{array}$ & 0,284 & $-0,031$ & 0,307 & 0,056 & $-0,040$ & 0,160 & 0,712 \\
\hline 33 & $\begin{array}{l}\text { Establezco las normas de un curso o actividad docente a } \\
\text { través del diálogo y/o la negociación con los estudiantes }\end{array}$ & 0,070 & $-0,104$ & 0,360 & 0,316 & $-0,014$ & 0,104 & 0,698 \\
\hline
\end{tabular}


Tabla 2. Continuación

\begin{tabular}{|c|c|c|c|c|c|c|c|c|}
\hline Ítem & Reactivo & $\mathbf{I}$ & II & III & IV & $\mathbf{v}$ & VI & Comunalidad \\
\hline 34 & Utilizo ejemplos de la vida cotidiana de los estudiantes & 0,473 & $-0,054$ & $-0,026$ & 0,206 & 0,073 & 0,037 & 0,675 \\
\hline 35 & $\begin{array}{l}\text { Realizo actividades dirigidas a motivar el aprendizaje de } \\
\text { una unidad o tema }\end{array}$ & 0,478 & 0,159 & 0,014 & 0,299 & $-0,144$ & $-0,161$ & 0,471 \\
\hline 36 & $\begin{array}{l}\text { Integro los intereses de mis estudiantes durante el desa- } \\
\text { rrollo de mis actividades docentes }\end{array}$ & 0,501 & 0,067 & 0,093 & 0,310 & 0,011 & $-0,253$ & 0,446 \\
\hline 37 & $\begin{array}{l}\text { Utilizo estrategias para captar la atención de los estu- } \\
\text { diantes }\end{array}$ & 0,393 & 0,155 & $-0,098$ & 0,447 & $-0,144$ & $-0,104$ & 0,467 \\
\hline 38 & $\begin{array}{l}\text { Realizo preguntas a los estudiantes (individuales o gru- } \\
\text { pales) para motivar el diálogo con ellos }\end{array}$ & 0,417 & $-0,158$ & 0,101 & 0,404 & 0,051 & 0,016 & 0,549 \\
\hline 39 & $\begin{array}{l}\text { Participo de reuniones con directivos de la carrera y } \\
\text { otros docentes para verificar los contenidos que se están } \\
\text { enseñando }\end{array}$ & 0,138 & 0,102 & 0,354 & 0,060 & 0,098 & 0,028 & 0,733 \\
\hline 40 & Promuevo el debate entre los estudiantes & 0,460 & $-0,058$ & 0,316 & 0,250 & $-0,006$ & $-0,064$ & 0,458 \\
\hline 42 & $\begin{array}{l}\text { Realizo conexiones explícitas entre lo que los estudiantes } \\
\text { aprenden en mis actividades y lo que pasa en el mundo } \\
\text { del trabajo }\end{array}$ & 0,566 & $-0,054$ & 0,036 & 0,194 & 0,030 & $-0,024$ & 0,573 \\
\hline 43 & $\begin{array}{l}\text { Organizo mis actividades docentes (clases, supervisiones, } \\
\text { etc.) puntualizando los momentos de una secuencia de } \\
\text { aprendizaje en aula (introducción, desarrollo y cierre) }\end{array}$ & 0,047 & 0,426 & 0,096 & 0,344 & 0,039 & $-0,056$ & 0,523 \\
\hline 44 & $\begin{array}{l}\text { Uso criterios dicotómicos para diferenciar si un estudian- } \\
\text { te sabe o no sabe, por ejemplo aprobado- reprobado }\end{array}$ & $-0,109$ & 0,092 & 0,292 & 0,032 & 0,386 & $-0,090$ & 0,753 \\
\hline 45 & $\begin{array}{l}\text { Limito la bibliografía a considerar por mis estudiantes a } \\
\text { la que yo definí previamente }\end{array}$ & 0,027 & $-0,028$ & 0,093 & 0,004 & 0,375 & $-0,043$ & 0,858 \\
\hline 46 & $\begin{array}{l}\text { Uso estrategias expositivas para abordar contenidos } \\
\text { complejos o de alto nivel de abstracción }\end{array}$ & $-0,011$ & $-0,010$ & $-0,135$ & 0,098 & 0,661 & 0,038 & 0,527 \\
\hline 47 & $\begin{array}{l}\text { Realizo evaluaciones diagnósticas al inicio de un curso } \\
\text { o unidad }\end{array}$ & 0,005 & 0,198 & 0,417 & 0,048 & 0,016 & $-0,078$ & 0,703 \\
\hline 48 & $\begin{array}{l}\text { Utilizo rúbricas de desempeño para evaluar a los estu- } \\
\text { diantes }\end{array}$ & $-0,113$ & 0,217 & 0,361 & 0,083 & $-0,081$ & 0,204 & 0,696 \\
\hline 49 & $\begin{array}{l}\text { Expreso expectativas positivas a mis estudiantes respecto } \\
\text { de sus logros }\end{array}$ & 0,102 & 0,152 & 0,061 & 0,526 & $-0,092$ & 0,120 & 0,517 \\
\hline 50 & $\begin{array}{l}\text { Uso estrategias que hagan participar activamente al } \\
\text { estudiante como aprendizaje basado en problemas, } \\
\text { aprendizaje por proyectos, aprendizaje y servicio, etc. }\end{array}$ & 0,333 & 0,059 & 0,331 & 0,111 & $-0,096$ & $-0,061$ & 0,614 \\
\hline 53 & Establezco relaciones cordiales con los estudiantes & $-0,021$ & 0,000 & $-0,085$ & 0,518 & 0,010 & 0,049 & 0,746 \\
\hline 54 & $\begin{array}{l}\text { Estimulo la argumentación en el discurso de los estu- } \\
\text { diantes }\end{array}$ & 0,150 & $-0,035$ & 0,166 & 0,501 & 0,107 & 0,034 & 0,582 \\
\hline 57 & $\begin{array}{l}\text { Utilizo medios audiovisuales para clarificar contenidos: } \\
\text { diapositivas videos, imágenes }\end{array}$ & $-0,062$ & 0,035 & $-0,044$ & 0,172 & 0,307 & 0,354 & 0,714 \\
\hline 58 & $\begin{array}{l}\text { Organizo mis actividades docentes (clases, supervisio- } \\
\text { nes, etc.) dependiendo de los avances que tengan los } \\
\text { estudiantes }\end{array}$ & $-0,149$ & 0,386 & 0,131 & 0,355 & 0,000 & $-0,052$ & 0,634 \\
\hline 59 & $\begin{array}{l}\text { Realizo recapitulaciones orales que monitorean la com- } \\
\text { prensión de los estudiantes (por ejemplo, repito aquellos } \\
\text { conceptos que deben quedar más claros, pregunto si } \\
\text { algo no se ha entendido) }\end{array}$ & 0,067 & 0,228 & $-0,057$ & 0,492 & $-0,027$ & 0,044 & 0,612 \\
\hline \multirow[t]{3}{*}{60} & $\begin{array}{l}\text { Utilizo el refuerzo social constante con mis estudiantes: } \\
\text { agradecimientos, felicitaciones públicas, etc. }\end{array}$ & 0,053 & 0,031 & 0,159 & 0,572 & 0,047 & 0,063 & 0,533 \\
\hline & Autovalores & 11,52 & 2,71 & 1,98 & 1,38 & 1,13 & 1,02 & \\
\hline & $\%$ de varianza total & 48,12 & 11,32 & 8,25 & 5,76 & 4,71 & 4,27 & \\
\hline
\end{tabular}

aLas cursivas indican cargas superiores a 0,30; (i)́tem inverso 
ítems presentaron cargas superiores a 0,30 en al menos un factor, con 10 casos (ítems 17, 33, 36, $38,37,40,43,50,57$ y 58 ) que las presentaban en dos factores simultáneamente (carga cruzada). En estas situaciones, los ítems fueron asignados considerando la coherencia teórica con cada factor.

Así, los factores quedaron conformados como sigue:

- Factor I: Se denominó Enseñanza centrada en el estudiante. Presentó un alfa de Cronbach de $\alpha=0,85$, con correlaciones entre los ítems y el total corregido entre $r=0,43$ (ítem 9) y $r=0,61$ (ítem 28).

- Factor II: Denominándose Planificación de la enseñanza, exhibió un alfa de $\alpha=0,85$, con correlaciones ítem-total corregido desde $r=0,44$ (ítem 2) hasta $r=0,65$ (ítem 8).

- Factor III: Se denominó Evaluación de proceso, y obtuvo un alfa de Cronbach de $\alpha=0,81$, con correlaciones ítem-total corregido desde $r=0,41$ (ítem 39) hasta $r=0,64$ (ítem 27).

- Factor IV: Se denominó Relación dialogante, y presentó un alfa de Cronbach de $\alpha=0,82$, con correlaciones ítem-total corregido desde $r=0,43$ (ítem 53) hasta $r=0,61$ (ítem 49).

- Factor V: Etiquetado como Enseñanza centrada en el profesor, presentó un alfa de Cronbach de $\alpha=0,64$, con correlaciones ítem-total corregido desde $r=0,27$ (ítem 44) hasta $r=0,51$ (ítem 46).

- Factor VI: Se denominó Uso de recursos tecnológicos, y tuvo un alfa de Cronbach de $\alpha=0,60$, con correlaciones ítem-total corregido desde $r=0,25$ (ítem 10) hasta $r=0,42$ (ítem 24).

La composición y definición conceptual de los factores se detalla en la Tabla 3. Luego se calculó sus puntajes sumando sus ítems, y se realizó un análisis descriptivo de cada uno. Para facilitar la interpretación de los puntajes de los factores, se sugieren tres niveles interpretativos: Nivel bajo (puntajes inferiores al percentil 25), Nivel medio (puntajes iguales o mayores al percentil $25 \mathrm{y}$ menores al percentil 75) y Nivel alto (puntajes iguales o mayores al percentil 75) (Tabla 4).

Tabla 3. Factores identificados para el cuestionario de prácticas pedagógicas: definición
conceptual e ítems incluidos

\begin{tabular}{|c|c|c|}
\hline Factor & Definición conceptual & $\begin{array}{l}\text { Ítems incluidos (de } \\
\text { mayor a menor carga) }\end{array}$ \\
\hline $\begin{array}{l}\text { 1. Enseñanza centrada } \\
\text { en el estudiante }\end{array}$ & $\begin{array}{l}\text { Actividades para buscan que el estudiante aprenda, caracte- } \\
\text { rizadas por una orientación constructivista con énfasis en el } \\
\text { desarrollo integral del estudiante y en una mayor participación } \\
\text { de éste en su proceso formativo }\end{array}$ & $\begin{array}{c}28,42,36,29,35,34 \\
40,17,19,38,37 \text { y } 9\end{array}$ \\
\hline $\begin{array}{l}\text { 2. Planificación de la } \\
\text { enseñanza }\end{array}$ & $\begin{array}{l}\text { Actividades de preparación que buscan generar una actividad } \\
\text { pedagógica ordenada y secuenciada, orientada a favorecer el } \\
\text { aprendizaje del estudiante }\end{array}$ & $\begin{array}{c}8,1,20,16,14,5 \\
43,2,58,13,22 \text { y } 7\end{array}$ \\
\hline 3. Evaluación de proceso & $\begin{array}{l}\text { Actividades que buscan realizar una evaluación del aprendizaje } \\
\text { del estudiante de manera continua, integral y desde múltiples } \\
\text { fuentes de información }\end{array}$ & $\begin{array}{c}12,27,30,47,48,33 \\
39,31,50 \text { у } 32\end{array}$ \\
\hline 4. Relación dialogante & $\begin{array}{l}\text { Actividades para establecer una relación cálida, empática, res- } \\
\text { petuosa y motivadora con los estudiantes para contextualizar } \\
\text { favorablemente su proceso formativo }\end{array}$ & $60,49,53,54,59$ у 21 \\
\hline $\begin{array}{l}\text { 5. Enseñanza centrada } \\
\text { en el profesor }\end{array}$ & $\begin{array}{l}\text { Actividades para buscan que el estudiante aprenda, caracteriza- } \\
\text { das por una orientación tradicional, centrada en el profesor y en } \\
\text { la transmisión de contenidos declarativos, y que promueve una } \\
\text { actitud pasiva del estudiante }\end{array}$ & $11,46,3,6,44$ y 45 \\
\hline $\begin{array}{l}\text { 6. Uso de recursos } \\
\text { tecnológicos }\end{array}$ & $\begin{array}{l}\text { Actividades para diseñar y emplear dispositivos y herramientas } \\
\text { tecnológicas para apoyar eficazmente el proceso formativo, es- } \\
\text { timulando a su vez, él uso de éstas por parte de los estudiantes }\end{array}$ & $24,10,18,23$ у 57 \\
\hline
\end{tabular}


Tabla 4. Estadísticos descriptivos de los factores del cuestionario de prácticas pedagógicas en docentes de carreras de la salud

\begin{tabular}{|lcccccc|}
\hline Estadísticos & $\begin{array}{c}\text { Enseñanza } \\
\text { centrada en } \\
\text { el estudiante }\end{array}$ & $\begin{array}{c}\text { Planificación } \\
\text { de la } \\
\text { enseñanza }\end{array}$ & $\begin{array}{c}\text { Evaluación } \\
\text { de proceso }\end{array}$ & $\begin{array}{c}\text { Relación } \\
\text { dialogante }\end{array}$ & $\begin{array}{c}\text { Enseñanza } \\
\text { centrada en } \\
\text { el profesor }\end{array}$ & $\begin{array}{c}\text { Uso de } \\
\text { recursos } \\
\text { tecnológicos }\end{array}$ \\
\hline Número de ítems & 12 & 11 & 10 & 6 & 6 & 5 \\
\hline Alfa de Cronbach & 0,85 & 0,85 & 0,81 & 0,82 & 0,64 & 0,60 \\
\hline Media aritmética & 32,79 & 38,44 & 27,42 & 28,12 & 14,32 & 16,97 \\
\hline Desviación estándar & 4,77 & 6,26 & 6,58 & 3,36 & 3,83 & 2,49 \\
\hline \% & 43,31 & 62,36 & 43,55 & 92,17 & 34,66 & 59,85 \\
\hline Percentil 25 & 29 & 34 & 24 & 26 & 12 & 16 \\
\hline Percentil 50 & 33 & 39 & 28 & 29 & 14 & 17 \\
\hline Percentil 75 & 37 & 43 & 32 & 31 & 17 & 19 \\
\hline Asimetría & $-0,31$ & $-0,78$ & $-0,59$ & $-0,99$ & $-0,19$ & $-1,01$ \\
\hline Curtosis & 2,39 & 3,73 & 3,34 & 4,05 & 3,31 & 4,09 \\
\hline N & 29 & & & 17 \\
\hline
\end{tabular}

$\mathrm{N}=296 ; \%=$ (Puntaje obtenido - Puntaje mínimo posible) / (Puntaje máximo posible - Puntaje mínimo posible)*100.

Tabla 5. Correlaciones de Pearson entre los factores del cuestionario de prácticas pedagógicas en docentes de carreras de la salud

\begin{tabular}{|c|c|c|c|c|c|c|}
\hline & 1 & 2 & 3 & 4 & 5 & 6 \\
\hline 1. Enseñanza centrada en el estudiante & $0,85^{a}$ & & & & & \\
\hline 2. Planificación de la enseñanza & $0,62^{* * *}$ & $0,85^{a}$ & & & & \\
\hline 3. Evaluación de proceso & $0,59 * * *$ & $0,64 * * *$ & $0,81^{a}$ & & & \\
\hline 4. Relación dialogante & $0,67 * * *$ & $0,60 * * *$ & $0,50 * * *$ & $0,82^{a}$ & & \\
\hline 5. Enseñanza centrada en el profesor & 0,10 & $0,14^{*}$ & $-0,02$ & 0,05 & $0,64^{a}$ & \\
\hline 6. Uso de recursos tecnológicos & $0,28 * * *$ & $0,35 * * *$ & $0,25 * * *$ & $0,27 * * *$ & $0,20 * * *$ & $0,60^{\mathrm{a}}$ \\
\hline
\end{tabular}

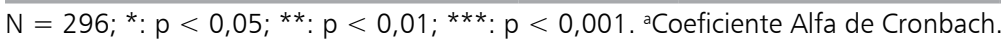

Por último, se analizó la correlación entre los factores del CPP, mostrando correlaciones directas y estadísticamente significativas entre todos ellos, con excepción de Enseñanza centrada en el profesor, que no mostró correlaciones en tres casos (Tabla 5).

\section{Discusión}

La práctica pedagógica es un fenómeno complejo, procesal y contextualmente determinado, que apunta a la reflexión constante que los académicos realizan sobre cada situación de enseñanza a fin de definir su curso de acción ${ }^{18-22}$, y se expresa finalmente en su forma de actuar dentro del escenario formativo. Es por este motivo que el CPP focaliza su evaluación de las prácticas pedagógicas en la frecuencia con que el docente realiza actividades específicas, referidas a las diversas funciones educativas que debe asumir, diferenciándose de otros instrumentos que evalúan qué autoeficacia pero la acción docente ${ }^{37}$, y de aquellos que evalúan la acción pero en temáticas específicas ${ }^{38}$.

$\mathrm{Al}$ respecto, los resultados muestran que el CPP mediría seis factores de las prácticas pedagógicas, que se sintetizarían en cinco roles del docente: planificar, enseñar, evaluar, gestionar el ambiente y desarrollar recursos educativos.

Primero, las actividades que realiza el docente 
para favorecer el aprendizaje del estudiante están presentes en dos factores, Enseñanza centrada en el estudiante y Enseñanza centrada en el profesor, los que reflejan los dos modelos formativos que en la actualidad se encuentran en pugna en la formación de las carreras de la salud, el llamado modelo tradicional, unidimensional, basado en la transmisión de conocimiento declarativo, en la hegemonía docente como fuente de conocimiento y en la pasividad del estudiante, y el modelo constructivista, que busca favorecer el aprendizaje en distintas áreas (cognitivas, afectivas, sociales, conductuales, etc.) a través de una mayor participación del estudiantado y una mayor vinculación de éste con su contexto ${ }^{8,15,30}$. Hay una crítica consensuada al primero de estos modelos, y las carreras de la salud buscan transitar al modelo centrado en los estudiantes $^{8}$. Sin embargo, el tradicional sigue siendo común en la universidad ${ }^{17}$ y es altamente eficiente para transmitir información ${ }^{34,35}$. En este sentido, que el CPP evalúe la frecuencia con que los docentes de carreras de la salud recurren a ambos modelos es pertinente para un escenario como el actual donde ambos están vigentes aún, siendo adecuados para situaciones de aprendizaje específicas. Asimismo, es útil diferenciarlos, dado que no constituirían dos polos de una dimensión, sino dos dimensiones independientes, como lo demuestra la ausencia de correlación identificada entre ambos.

Segundo, el CPP evalúa el grado de Planificación de la enseñanza, incluyendo tanto las acciones tendientes a organizar previamente la acción pedagógica como al uso de recursos y dispositivos para facilitar este proceso (p.e. programas, syllabus, etc.). Asimismo, considera la planificación semestral y la definición de los momentos de la clase.

Tercero, se identificó un único factor sobre evaluación: Evaluación de proceso, que rescata una función inherente a la docencia ${ }^{11,14,15}$ : la constatación de avances en el aprendizaje de los estudiantes. El modelo evaluativo que rescata, integrando modalidades sumativas y formativas, heteroevaluativas y autoevaluativas, está alineado con los modelos curriculares que se promueven en la actualidad en carreras de la salud ${ }^{8}$.

El cuarto, la Relación dialógica, aborda la capacidad del docente para generar espacios de aprendizaje propicios, motivadores, empáticos y respetuosos ${ }^{11}$. Aunque parece trascender a los aspectos disciplinares tradicionalmente asociados a los factores anteriores, refiere a una competencia básica que afecta directamente los aprendizajes alcanzados por los estudiantes y la capacidad misma del docente para actuar como modelo conductual ${ }^{15}$.

Finalmente, el Uso de recursos tecnológicos ya era un rol reconocido explícitamente por Harden, al hablar del creador de recursos materiales ${ }^{14,15}$, y aborda un aspecto esencial para nuestra sociedad tecnologizada.

Ante esto, la evaluación que hace el CPP, centrada en el comportamiento docente, no desconoce los aspectos que afectan las práctica pedagógica, tanto personales, de docentes y estudiantes ${ }^{19,20}$, como ambientales, del aula, el currículum formal y oculto ${ }^{21}$. Sin embargo, para operacionalizar el constructo de las prácticas y facilitar su medición, se enfoca en una dimensión de éste: la conducta docente, bajo la convicción que los otros factores, aunque relevantes, requieren mediciones diferenciadas.

Por otra parte, el CPP presenta una adecuada confiabilidad en cuatro de sus factores, pero esta es cuestionable en los otros dos, lo que puede deberse a su menor número de ítems ${ }^{31,36}$. Sin embargo, es posible utilizarlo para el diagnóstico y la investigación de esta variable, aunque no en evaluaciones con consecuencias directas para los estudiantes (selección, evaluación sumativa, etc.).

En síntesis, en este estudio se ha obtenido evidencia de la validez y confiabilidad del CPP para medir prácticas pedagógicas en docentes de carreras de la salud de Chile.

El presente estudio tuvo un alcance nacional, no obstante la estrategia de muestreo por voluntarios dificultó una muestra más representativa de algunas regiones. Por otra parte, dado que las prácticas pedagógicas presentan una alta deseabilidad social, el CPP puede hacer un tamizaje eficiente y a bajo costo. Sin embargo, cuando sea posible es recomendable complementarlo con otras estrategias de recolección de datos, como observación en aula.

\section{Referencias}

1. Tan $\mathrm{CH}$, Macneill P. Globalization, economics and professionalism. Med Teach 2015; 37 (9): 850-5.

2. Fernández E, Bernasconi A. Las nuevas carreras de medicina como símbolo: entre la legitimidad académica y 
la señalización de mercado. Education Policy Analysis Archives 2014; 22: 1-17.

3. Wilkerson L, Irby DM. Strategies for improving teaching practices: a comprehensive approach to faculty development. Acad Med 1998; 73 (4): 387-96.

4. Castillo M, Hawes G, Castillo S, Romero L, Rojas AM, Espinoza M, et al. Cambio educativo en las Facultades de Medicina. Rev Med Chile 2014; 142 (8): 1056-60.

5. Pérez C, Parra P, Ortiz L, Fasce E. Variables personales y académicas asociadas al aprendizaje autodirigido en la educación médica. Rev Educ Cienc Salud 2010; 7 (2): 152-9.

6. Spormann C, Pérez C, Fasce E, Ortega J, Bastías N, Bustamante $\mathrm{C}$, et al. Predictores afectivos y académicos del aprendizaje autodirigido en estudiantes de medicina. Rev Med Chile 2015; 143 (3): 374-82.

7. Parada M, Romero MI, Moraga F. Perfiles de egreso de las carreras de medicina en Chile. Rev Med Chile 2015; 143 (4): 512-9.

8. Fasce E. ¿Son suficientes las competencias profesionales en la enseñanza de las ciencias de la salud? Rev Educ Cienc Salud 2008; 5 (2): 81.

9. Martínez A, Amaro R. El docente universitario y su espacio de formación. Fundamentación de una propuesta. Docencia universitaria 2008; 9 (2): 53-80.

10. Steinert Y, Mann K, Centeno A, Dolmans D, Spencer J, Gelula M, et al. A systematic review of faculty development initiatives designed to improve teaching effectiveness in medical education: BEME Guide No 8 . Med Teach 2006; 28 (6): 497-526.

11. Tejedor FJ. Evaluación del profesorado universitario: enfoque metodológico y algunas aportaciones de la investigación. Estudios sobre educación 2009; 16: 79-102.

12. Zabalza MA. La convergencia como oportunidad para mejorar la docencia universitaria. Revista universitaria de formación del profesorado 2006; 20 (3): 37-69.

13. Álvarez-Rojo V, Asensio-Muñoz I, Clares J, del-Frago R, García-Lupión B, García-Nieto N, et al. Perfiles docentes para el espacio europeo de educación superior (EEES) en el ámbito universitario español. RELIEVE 2009; 15 (1): 1-18.

14. Srinivasan M, Li S, Meyers F, Pratt D, Collins J, Braddock $\mathrm{C}$, et al. "Teaching as a compentency": Competencies for medical educator. Acad Med 2011; 86 (10): 1211-20.

15. Harden R, Crosby R. The good teacher is more than a lecturer-the twelve roles of the teacher. AMEE Guide $\mathrm{N}^{\mathrm{o}}$ 20. Med Teach 2000; 22 (4): 334-47.

16. Fook CY. Best practices of teaching in higher education in United States: a case study. Procedia, Social and behavioral sciences 2012; 40: 4817-21.
17. Morales I, Borroto R, Fernández B. Políticas y estrategias para la transformación de la educación superior en América Latina y el Caribe. Educ Méd Super 2005; 19 (1): 1-1.

18. Cañedo T, Figueroa A. La práctica docente en educación superior: una mirada hacia su complejidad. Sinéctica 2013; 41: 2-18.

19. Juliá M. Psicología Educacional. Proponiendo rumbos, Problemáticas y Aportaciones. Santiago de Chile: Universidad de la Serena; 2011.

20. Rueda M. La evaluación del desempeño docente desde el enfoque por competencias. Revista electrónica de investigación educativa 2009; 11 (2): 1-12.

21. Díaz V. Formación docente, práctica pedagógica y saber pedagógico. Laurus 2006; 12 (Ext): 88-110.

22. Barrón MA. Docencia universitaria y competencias didácticas. Perfiles educativos 2009; 31 (125): 76-87.

23. Triviño X, Sirhan M, Moore P, Montero L. Impacto de un programa de formación en docencia en una escuela de medicina. Rev Med Chile 2011; 139: 1508-15.

24. Montero L, Triviño X, Sirhan M, Moore P, Leiva L. Barreras para la formación en docencia de los profesores de medicina: una aproximación cualitativa. Rev Med Chile 2012, 140: 695-702.

25. Sirhan M, Triviño X. Evaluación de una experiencia de capacitación en planificación educacional para directores de programas de las especialidades médicas. Rev Med Chile 2012; 140: 530-7.

26. Pérez C, Fasce E, Coloma K, Vaccarezza G, Ortega J. Percepción de académicos de carreras de la salud de Chile sobre el perfeccionamiento docente. Rev Med Chile 2013; 141: 787-92.

27. Arámburo V, Luna E. La influencia de las características, profesor y del curso en los puntajes de evaluación docente. Revista mexicana de investigación educativa 2013; 18 (58): 949-68.

28. Abello D, Hernández C, Hederich C. Estilos de enseñanza en docentes universitarios, propuesta y validación de un modelo teórico e instrumental. Pedagogía y saberes 2011; 34: 141-54.

29. Casero A. Propuesta de un cuestionario de evaluación de la calidad docente universitaria consensuado entre alumnos y profesores. Revista de Investigación educativa 2008; 26: 25-44.

30. Snider V, Roehl R. Teachers' beliefs about pedagogy and related issues. Psychology in the schools 2007; 44 (8): 873-86.

31. Martínez R, Hernández MJ, Hernández MV. Psicometría. Madrid: Alianza; 2006.

32. Tabachnick BG, Fidell LS. Using multivariate statistics. New York: Harper and Row; 2006. 
33. Hair JF, Black WX, Babin BJ, Anderson RE, Tatham RL. Análisis multivariante. Madrid: Prentice Hall; 2005.

34. Coppola B. The distinctiveness of higher education. Journal of chemical education 2013; 90: 955-6.

35. Flechsig KH, Schiefelbein E. 20 modelos didácticos para América Latina. Washington: Organización de los Estados Americanos; 2003.

36. George D, Mallery P. SPSS for Windows step by step:
A simple guide and reference. 11.0 update (4th ed.). Boston: Allyn \& Bacon; 2003.

37. Fernández M, Merino C. Resultados psicométricos preliminares de la escala de autoeficacia percibida en maestros de Lima. Psicogente 2012; 15: 314-22.

38. Barahona E. Estudio de Validez del Cuestionario de Prácticas Pedagógicas Para la Creatividad (CPPC). Psykhe 2004; 13: 157-74. 